03

\title{
Звуковой удар от тонкого тела и локальных областей нагрева сверхзвукового набегающего потока
}

\author{
(C) А.В. Потапкин, Д.Ю. Москвичев \\ Институт теоретической и прикладной механики им. С.А. Христиановича СО РАН, \\ 630090 Новосибирск, Россия \\ e-mail: potapkin@itam.nsc.ru
}

Поступило в Редакцию 16 августа 2020 г.

В окончательной редакции 15 октября 2020 г.

Принято к публикации 17 октября 2020 г.

\begin{abstract}
Численно решалась задача о звуковом ударе от тонкого тела и локальных областей нагрева сверхзвукового набегающего потока. Число Маха набегающего потока воздуха равно 2. Расчеты выполнены с помощью комбинированного метода „тел-фантомов“. Результаты расчетов показали, что локальный разогрев набегающего потока может обеспечить снижение уровня звукового удара. Уровень звукового удара зависит от количества локальных областей нагрева набегающего потока. При одной области нагрева потока можно получить снижение уровня звукового удара на 20\%, по сравнению с уровнем звукового удара от тела в холодном потоке. А последовательный нагрев набегающего потока в двух областях нагрева обеспечивает уменьшение уровня звукового удара более чем на $30 \%$.
\end{abstract}

Ключевые слова: сверхзвуковой полет, ударные волны, звуковой удар, тонкое тело, нагрев потока, метод „тел-фантомов“.

DOI: $10.21883 /$ JTF.2021.04.50618.248-20

\section{Введение}

В настоящее время разрабатываются различные способы снижения уровня звукового удара от сверхзвукового самолета с помощью разогрева набегающего потока. Примером является работа [1], в которой рассмотрен способ снижения звукового удара от сверхзвукового самолета с помощью разогрева атмосферы вокруг самолета лазерным излучением. Разогрев атмосферы вокруг самолета в локальной области определенной формы с контролируемым распределением температуры в области должен предотвратить распространение возмущений от летательного аппарата за границы этой области, что может обеспечить снижение уровня звукового удара. В работах [2,3] разработана аналитическая линейная теория сверхзвукового обтекания тонких тел вращения при воздействии внешнего подвода энергии и внешней силы на поток в тонком приповерхностном слое обтекаемого тела. Авторы предлагают использовать эту теорию для решения оптимизационных задач, например, снижения лобового сопротивления и уменьшения уровня акустического шума в ходе сверхзвукового обтекания при минимальных энергозатратах. В результате анализа сделан вывод, что использование энергетического и силового воздействий на сверхзвуковой поток в тонком слое около обтекаемого тела позволяет ослабить акустический шум, генерируемый телом вращения. В патенте [4] предлагалось использовать подвод лучистой энергии от источника лазерного излучения к набегающему потоку воздуха вдоль передней кромки крыла самолета для обеспечения дозвукового обтекания крыла и уменьшения звукового удара в сверхзвуковом полете.
Уменьшение интенсивности ударных волн в ближнем поле при разогреве набегающего потока воздуха с помощью пульсирующего подвода энергии от лазерного излучения экспериментально и теоретически изучалось в работе [5]. Результаты исследований показали, что разогрев потока около тела может быть использован для снижения интенсивности ударных волн от тела в ближнем поле. Расширенный обзор статей по проблеме звукового удара можно найти в работе [6]. Необходимо отметить, что в большинстве работ не принимаются во внимание ударные волны от области нагрева потока и их роль в формировании звукового удара в дальнем поле. Целью настоящей работы является исследование влияния локального нагрева набегающего потока на динамику ударных волн и уровень звукового удара от тонкого тела вращения. Изучается возможность уменьшения уровня звукового удара при различных способах разогрева набегающего потока.

При исследовании звукового удара необходимо учитывать большое количество факторов, определяющих динамику ударных волн. Измерения на больших расстояниях от тела в аэродинамических трубах с локальным нагревом сверхзвукового потока выполнить невозможно из-за малых размеров рабочей части аэродинамических труб. К настоящему времени натурные эксперименты и эксперименты в баллистических трассах с нагревом сверхзвукового потока возле самолета не проводились. Поэтому основными инструментами для решения этих задач являются аналитические и численные методы. Можно выделить два способа расчета звукового удара в дальнем поле. Первый способ (комбинированный) заключается в определении параметров возмущенного по- 
тока в ближнем поле летательного аппарата с помощью аналитических или численных методов с последующим пересчетом этих параметров в дальнее поле [7-9]. Второй способ (прямой) заключается в численном расчете параметров звукового удара для всего поля течения [10].

Для расчета параметров слабых ударных волн на больших расстояниях от места их возникновения разработан комбинированный численно-аналитический метод, который получил название „тел-фантомов“ [9]. Расчеты звукового удара основаны на последовательном вычислении параметров ближнего и дальнего поля с использованием аналитических решений Ландау [11], Уизема [12,13] и Рао [14]. Метод можно использовать для вычисления звукового удара как от одного тела, так и от группы тел.

\section{1. Описание метода „тел-фантомов“}

Схема метода для течения $\mathrm{c}$ осевой симметрией показана на рис. 1 для одного тела. Параметры ближнего поля (профиль избыточного давления 4) использовались для построения „тела-фантома“ 6 . „Тело-фантом“ дискретная последовательность точек на траектории полета. Координаты точек и значения функции Уизема в этих точках вычислялись по параметрам ближнего поля. В ближнем поле течения профиль давления 4 задавался в виде зависимости $\Delta P^{n}=\Delta P(x, r)$. Параметры ближнего поля приводились к безразмерному виду. Безразмерные параметры течения в ближнем поле вычислялись по формулам $p^{*}=p / p_{m}, \rho^{*}=\rho / \rho_{m}$, $a^{*}=a / u_{m}, L^{*}=L / L_{m}$. Здесь $p_{m}, \rho_{m}, u_{m}, L_{m}-$ соответствующие масштабные коэффициенты для давления, плотности, скорости потока и расстояния, $a-$ скорость звука. Масштабные коэффициенты $p_{m}=p_{0}, \rho_{m}=\rho_{0} / \gamma$, $u_{m}=a_{0}, L_{m}=L$ выбраны таким образом, что $p_{0}^{*}=1$, $\rho_{0}^{*}=\gamma, a_{0}^{*}=1, L^{*}=1, \gamma-$ показатель адиабаты. Нижний индекс „0“ указывает на параметры набегающего потока. В качестве масштабного коэффициента для времени задавался интервал времени, в течение которого тело преодолевает расстояние, равное своей длине при числе Маха, равном единице. Для таких масштабных коэффициентов уравнения в теории Уизема имеют одинаковую запись в размерных и безразмерных величинах, и далее уравнения выписываются без верхнего индекса „*“. При расчетах дальнего поля количество точек „тела-фантома“ зависит от структуры течения, т.е. от числа скачков в потоке, от градиентов волн разрежения и т.п. В расчетах использовалось 100 и более точек. Для расчетов с тремя и большим количеством скачков использовалось 250 и более точек на профиле давления. Продольные координаты „тела-фантома“ $l_{1}, l_{2} \ldots l_{k}$ вычислялись с помощью линейных соотношений геометрической акустики. Соответствующие значения функции Уизема $F_{i}=F\left(l_{i}\right)$ вычислялись по известным значениям в точках профиля избыточного давления ближнего поля

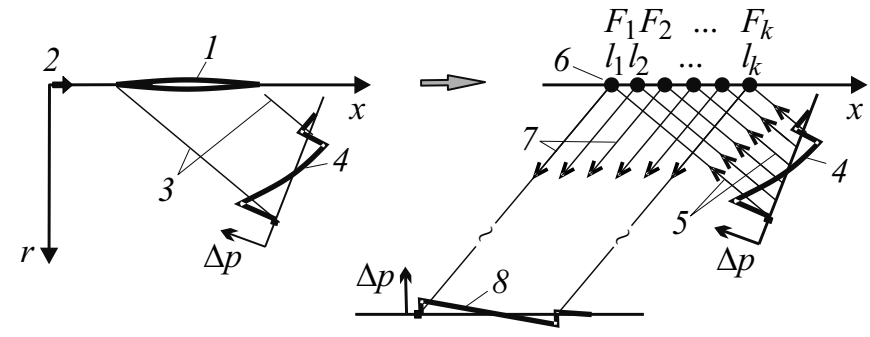

Рис. 1. Схема метода „тел-фантомов“: $1-$ тело, $2-$ направление набегающего потока, 3 - головной и замыкающий скачки в поле течения, 4 - профиль избыточного давления в ближнем поле, 5 - линейные характеристики, $6-$ „телофантом“, 7 - акустические лучи, 8 - профиль избыточного давления в дальнем поле.

по формуле

$$
F_{i}=\frac{\Delta P_{i}^{n}}{\rho_{0}} \cdot \sqrt{2} a_{0}^{-5 / 2} \mathrm{M}_{0}^{-3}\left[a_{0} \mathrm{M}_{0} \beta^{2} s_{i}^{n}\right]^{1 / 2},
$$

где $\mathrm{M}_{0}$ - число Маха набегающего потока, $\beta^{2}=\mathrm{M}_{0}^{2}-1, \quad r_{i}^{n}-$ радиальные координаты точек профиля давления $4, s^{n}-$ расстояние от точек профиля давления 4 до траектории полета вдоль акустических лучей. Связь между $r_{i}^{n}$ и $s_{i}^{n}$ определяется соотношением геометрической акустики $s_{i}^{n}=r_{i}^{n} \cdot \mathrm{M}_{0} / \beta$. Функция Уизема написана в соответствии с работой Рао [14] в предположении, что тело совершает полет с постоянной скоростью. Это удобно для вычислений и уравнение совпадает с уравнением (69) в работе Уизема [12].

Функция Уизема позволяет вычислить профиль давления для „тела-фантома“ в дальнем поле. Нелинейное уравнение характеристик $[13,14]$ может быть записано в виде $t-s_{i} / a_{0}+k F\left(\xi_{i}\right) b\left(s_{i}\right)=\xi_{i} / U(t)$. В этом выражении $t$ - время, $U(t)$ - мгновенная скорость полета тела, $k=(\gamma+1) \mathrm{M}_{0}^{5 / 2} /\left\{\sqrt{8} \beta a_{0}\right\}, b\left(s_{i}\right)=2 \sqrt{s_{i}}$. Значения характеристик $\xi_{i}$ определяются соотношением $\xi_{i}=l_{i}-\beta \cdot \tilde{r}_{i}$, где $l_{i}, \tilde{r}_{i}-$ координаты поверхности тела. При расчетах звукового удара можно использовать $\tilde{r}_{i}=0$. Это не вносит существенных ошибок в результаты расчетов. В нашем случае это единственный способ однозначного задания значений характеристик для „тела-фантома“. При таком упрощении уравнение характеристик записывается в виде

$$
t-s_{i} / a_{0}+k F\left(l_{i}\right) b\left(s_{i}\right)=l_{i} /\left(a_{0} \mathrm{M}_{0}\right) .
$$

Для заданного момента времени $t$ уравнение (2) позволяет найти $s_{i}$ - расстояния вдоль акустических лучей от точек тела фантома до точек профиля давления в дальнем поле.

Для вычисления давления в дальнем поле уравнение (1) может быть переписано в виде

$$
\Delta P_{i}=\frac{\rho_{0} F\left(l_{i}\right)}{\sqrt{2} a_{0}^{-5 / 2} \mathrm{M}_{0}^{-3} \cdot\left[a_{0} \mathrm{M}_{0} \beta^{2} s_{i}\right]^{1 / 2}} .
$$




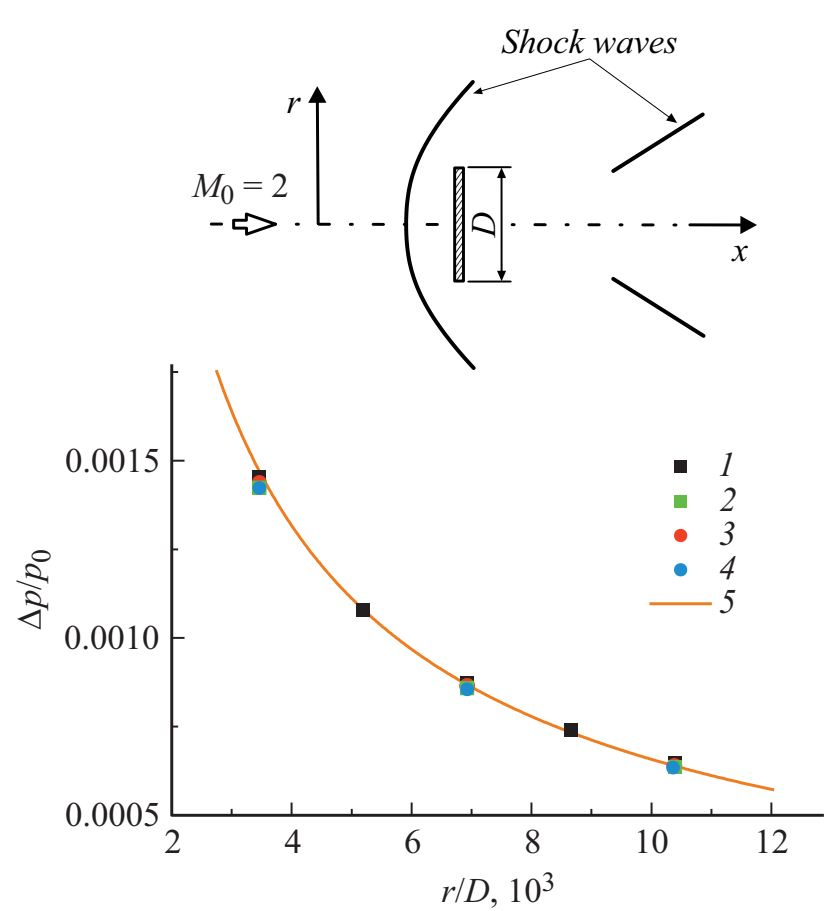

Рис. 2. Результаты расчетов дальнего поля по методу „телфантомов“ для различных начальных данных: $1-x_{0} / D=50$, $2-x_{0} / D=100,3-r_{0} / D=30,4-r_{0} / D=60,5-$ зависимость $\Delta p / p_{0} \sim(r / D)^{-3 / 4}[11]$.

Уравнение (3) позволяет построить профиль избыточного давления. Из-за нелинейности уравнения (2) при построении профиля давления вдоль акустического луча профиль давления опрокидывается и получается многозначное решение для давления. Неоднозначность решения для давления устранялась в соответствии с правилом Ландау [11] при помощи функции избыточного давления [15]. Результатом таких вычислений является профиль давления 8. Подробное описание дано в работах $[9,16,17]$, в которых метод тел фантомов использовался для расчета параметров звукового удара от тонких тел вращения, тел пространственной формы и затупленных тел.

Таким образом, с помощью этого метода производилась замена источников ударных волн на „тело-фантом“, для которого выполнялся расчет дальнего поля. Использование „тела-фантома“ позволяет понизить размерность решаемой задачи и рассчитывать дальнее поле течения с помощью теории Уизема с явным выделением фронтов ударных волн.

\section{2. Тестирование метода „тел-фантомов“}

Тестирование метода „тел-фантомов“ выполнено в работах $[9,16,17]$. Выполнены тестовые расчеты динамики ударных волн от тонкого заостренного тела вращения для $\mathrm{M}_{0}=2.4$ и затупленных тел для $\mathrm{M}_{0}=1.8$ и 2.0 .
Профили давления в ближнем поле вычислялись с помощью уравнений Эйлера или с помощью уравнений Навье-Стокса. Поверхность тонкого тела задавалась формулой $\tilde{r}=0.2 \cdot\left(x-x^{2} / L\right)$, где $\tilde{r}$ радиальная координата образующей поверхности тела, $L-$ длина тела, $0 \leq x \leq L$. Затупленное тело - шар или тонкий диск, расположенный поперек набегающего потока. Расчеты поля течения выполнялись в декартовой или цилиндрической системе координат. При расчетах трехмерного течения ближнего поля использовалась декартовая система координат $\{x, y, z\}: x-$ продольная координата, отсчитываемая от носика тела вниз по потоку, $y-$ вертикальная координата, $\{x y\}-$ плоскость симметрии, $z$ - боковое удаление от плоскости симметрии. При выполнении расчетов ближнего поля течения, имеющего осевую симметрию, использовалась цилиндрическая система координат $\{x, r\}: x-$ продольная координата, отсчитываемая от носика тела вниз по потоку, $r$ радиальная координата.

На рис. 2 показана схема течения вблизи затупленного тела и результаты расчетов дальнего поля при различном выборе начальных данных в расчетах звукового удара по методу „тел-фантомов“. Расчеты выполнялись для тонкого диска, расположенного поперек набегающего сверхзвукового потока воздуха. Число Маха набегающего потока $\mathrm{M}_{0}=2$. Обозначено $\Delta p-$ интенсивность головной ударной волны, $p_{0}$ - статическое давление в набегающем потоке, $r$ - расстояние от траектории полета, $D$ - диаметр диска. Профиль избыточного давления (начальные данные) для построения „тела-фантома“ выбирался либо как распределение давления $\Delta P(r)$ при фиксированном $x=x_{0}$, либо как $\Delta P(x)$ при фиксированном $r=r_{0}$. Здесь $x_{0}-$ расстояние вниз по потоку, $r_{0}-$ радиальное расстояние от траектории полета. На рисунке показана зависимость $\Delta p / p_{0} \sim(r / D)^{-3 / 4}[11]$, построенная по вычисленным значениям интенсивности головной ударной волны в дальнем поле.

При выборе начальных данных разными способами взаимный разброс результатов расчетов параметров дальнего поля не превышает $2 \%$, т.е. результаты расчетов по методу „тел-фантомов“ слабо зависят от способа построения начальных данных.

На рис. 3 показаны схемы течения и результаты тестовых расчетов для заостренного тонкого тела и шара. Обозначено $\Delta p^{\prime}$ - избыточное давление на мерной пластине за точкой отражения головной ударной волны, $D$ - диаметр шара, $h$ - расстояние от траектории полета до мерной пластины. Сравнение результатов расчетов для тонкого заостренного тела с аналитическими решениями и результатами расчетов, выполненных с помощью других методов, представлено на рис. 3, a. Линией 1 показан асимптотический закон изменения избыточного давления $\Delta p / p_{0} \sim(r / L)^{-3 / 4}$ в головной ударной волне от тонкого тела вращения при удалении от траектории полета на большие расстояния [11]. Точками 2 представлены результаты расчета звукового удара 


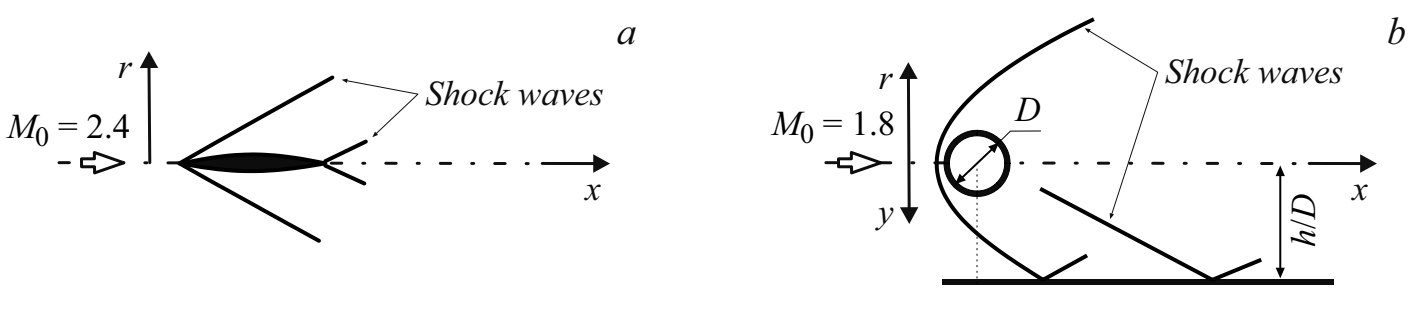

$b$
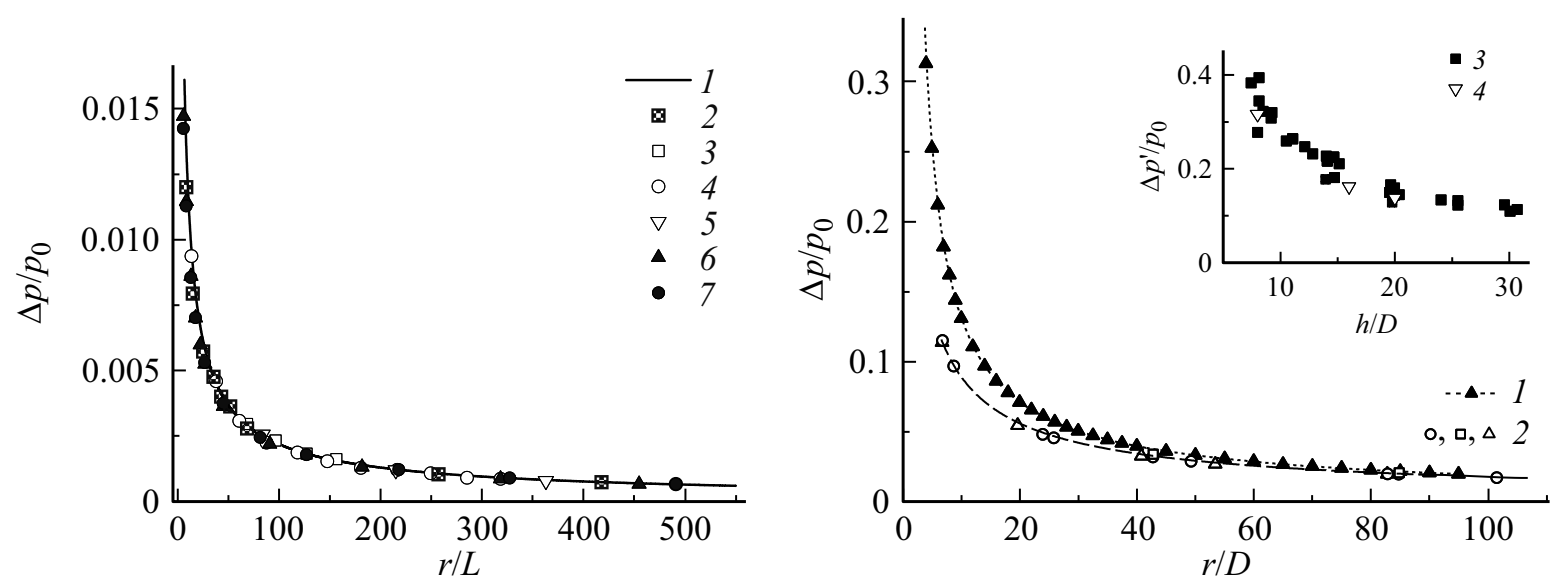

Рис. 3. Тестирование метода „тел-фантомов“. $a$ - заостренное тело вращения: 1 - зависимость $\Delta p / p_{0} \sim(r / L)^{-3 / 4}[11], 2-$ pacчеты по теории Уизема [12], 3-5 - расчеты динамики ударных волн в подвижных разностных сетках с использованием уравнений Эйлера при различных способах вычисления газодинамических параметров на границах подвижной расчетной области [8], 6,7 расчеты по методу „тел-фантомов“ с начальными данными, взятыми на разных расстояниях от тела [9]. $b-$ затупленное тело: 1 - расчеты интенсивности головной ударной волны с использованием уравнений Навье-Стокса, 2 - расчеты по методу „телфантомов“ с начальными данными, взятыми на разных расстояниях от шара [17], 3 - эксперименты в баллистической трассе [19], 4 - расчеты избыточного давления на мерной пластине за точкой отражения головной ударной волны с использованием уравнений Навье-Стокса.

от тонкого тела вращения, полученные с помощью теории Уизема [12]. Точками 3-5 показана интенсивность головной ударной волны от тонкого тела, вычисленная с помощью двумерных нестационарных уравнений Эйлера в подвижных разностных сетках. Расчеты выполнены по методу Годунова [18]. Результаты получены при различных способах вычисления газодинамических параметров на границах подвижной расчетной области. Расширенное описание алгоритмов вычислений представлено в работе [8]. Точками 6 и 7 показаны результаты вычислений интенсивности головной ударной волны от тонкого тела вращения, полученные с помощью метода „телфантомов“ с начальными данными, взятыми на расстояниях от носика тела $x_{0} / L=2.5$ и 8.0 соответственно. Разброс результатов расчетов на больших расстояниях не превышает $2 \%$.

На рис. 3, $b$ показано сравнение результатов расчетов с результатами экспериментальных исследований звукового удара от шаров разного диаметра в баллистических трассах [19]. Точками и пунктирной линией 1 показаны результаты расчетов интенсивности головной ударной волны с помощью двумерных осесимметричных уравнений Навье-Стокса. Точками 2 показаны результаты расчетов по методу „тел-фантомов“, полученные для начальных данных, которые были выбраны на расстоя- ниях $x_{0} / D=2.5$ и 5.0 от шара. Линия, проходящая по точкам 2 - средняя линия для результатов расчетов по методу „тел-фантомов“. Разброс результатов расчетов относительно средней линии не превышает $2.5 \%$. Точками 3 показаны результаты измерения перепада давления на мерной пластине в точке падения головной ударной волны [19]. Точками 4 показаны результаты трехмерных расчетов перепада давления на пластине за отраженной головной ударной волной, выполненные с использованием уравнений Навье-Стокса. Сближение линий показывает, что с расстояний $r / D \sim 40-60$ метод „тел-фантомов“ дает физически правильные результаты. В экспериментах с шарами различного диаметра [19] на этих расстояниях было зафиксировано, что эпюра давления принимает форму правильной $N$-волны. Сходимость решений при удалении от тела показывает возможность использования метода „тел-фантомов“ для расчета звукового удара от затупленных тел. Подробное описание метода „тел-фантомов“ для случая затупленных тел дано в работе [17].

Результаты тестовых расчетов показывают, что метод „тел-фантомов“ можно использовать в вычислениях параметров ударных волн в дальнем поле от заостренных и затупленных тел. 


\section{3. Звуковой удар при локальном нагреве набегающего потока}

При локальном нагреве потока в окрестности нагретой области формируется ударная волна. За областью нагрева развивается тепловой след с повышенной температурой, низкой плотностью и пониженными значениями чисел Маха. На рис. 4 показаны схемы течения и поле плотности потока вблизи локальных областей нагрева сверхзвукового набегающего потока воздуха $Q 1$, $Q 2$ и тонкого тела. Для этих схем течения выполнены расчеты ближнего и дальнего полей течения. Расчеты ближнего поля течения проводились с помощью пакета программ ANSYS Fluent с использованием модели вязкого теплопроводного газа без явного выделения фронтов ударных волн. Число Маха набегающего потока воздуха $\mathrm{M}_{0}=2$. Длина тонкого тела в расчетах задавалась $L=1 \mathrm{~m}$. Параметры набегающего потока воздуха $T_{0}=300 \mathrm{~K}, p_{0}=101325 \mathrm{~Pa}$. Расчеты течения в области нагрева, в тепловом следе и ближнем поле выполнялись при постоянных удельных теплоемкостях и показателе адиабаты $\gamma=1.4$. Для моделирования энерговыделения в области нагрева в правую часть уравнения энергии вводился источниковый член. Удельная мощность источника энерговыделения $q_{d}$ задавалась постоянной величиной в области нагрева. Расчеты выполнялись для областей нагрева $Q 1$ и $Q 2$ в форме шара. При выполнении расчетов варьировались следующие параметры: количество областей нагрева, размер области нагрева (радиус области $r_{d}$ ), расстояние между областью нагрева и тонким телом вращения $x_{d}$, удельная мощность источника энерговыделения $q_{d}$. Различные варианты расчетов и соответствующие безразмерные
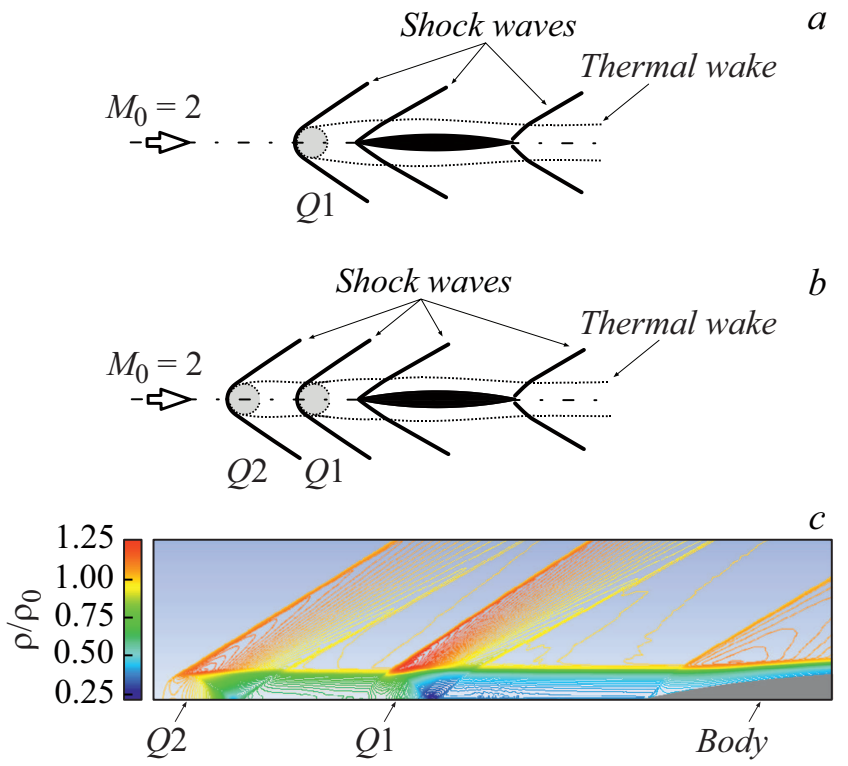

Рис. 4. $a, b-$ схемы течения, $c-$ пример поля плотности потока вблизи тела с двумя областями нагрева (изолинии плотности). $Q 1, Q 2$ - области нагрева сверхзвукового набегающего потока воздуха. наибольшие температуры воздуха в областях нагрева $T_{m}$ представлены в таблице.

Варианты расчетов

\begin{tabular}{c|c|l|l|c|c}
\hline $\begin{array}{c}\text { Число областей } \\
\text { нагрева }\end{array}$ & $\begin{array}{c}\text { Область } \\
\text { нагрева }\end{array}$ & $r_{d}, \mathrm{~m}$ & $x_{d}, \mathrm{~m}$ & $q_{d}, \mathrm{~W} / \mathrm{m}^{3}$ & $T_{m} / T_{0}$ \\
\hline 1 & $Q 1$ & 0.05 & 0.5 & $1.75 \cdot 10^{9}$ & 1.93 \\
\hline 1 & $Q 1$ & 0.05 & 0.0825 & $1.75 \cdot 10^{9}$ & 1.93 \\
\hline 1 & $Q 1$ & 0.05 & 0.175 & $2.625 \cdot 10^{9}$ & 2.41 \\
\hline 1 & $Q 1$ & 0.10 & 0.175 & $0.875 \cdot 10^{9}$ & 1.93 \\
\hline \multirow{2}{*}{2} & $Q 1$ & 0.05 & 0.5 & $1.75 \cdot 10^{9}$ & 2.80 \\
\cline { 2 - 6 } & $Q 2$ & 0.05 & 0.9 & $1.20 \cdot 10^{9}$ & 1.63 \\
\hline \multirow{2}{*}{2} & $Q 1$ & 0.05 & 0.5 & $1.90 \cdot 10^{9}$ & 2.92 \\
\cline { 2 - 6 } & $Q 2$ & 0.05 & 0.9 & $1.20 \cdot 10^{9}$ & 1.63
\end{tabular}

Параметры ближнего поля приводились к безразмерному виду. Результаты расчетов ближнего поля использовались в качестве начальных данных для расчетов дальнего поля с помощью метода „тел-фантомов““

\section{1. Разогрев набегающего потока в одной области перед телом}

Результаты расчетов с одной областью нагрева потока $Q 1$ представлены на рис. 5. На рис. 5, а показаны профили относительного избыточного давления $\Delta p / p_{0}$ вдоль продольной координаты $x$ на расстоянии $r / L=17$ от траектории полета. Значения относительной интенсивности $\Delta p / p_{0}$ ударных волн при удалении от траектории полета показаны на рис. $5, b$. Сплошными точками показаны интенсивности ударной волны от области нагрева, пустыми точками - интенсивности ударной волны от тела, находящегося в тепловом следе за областью нагрева. Линия 1 показывает интенсивность головной ударной волны от тела в холодном потоке воздуха. Линии 2 показывают процесс слияния ударных волн от области нагрева и тела, в результате чего интенсивность слившейся ударной волны приводит к увеличению уровня звукового удара. Линии $3-5$ показывают раздельное распространение ударных волн от области нагрева и тела. Линия $3^{\prime}$ показывает интенсивность ударной волны от области нагрева с $r_{d} / L=0.05$ и $T_{m} / T_{0}=1.93$ (без тела).

Разогрев потока воздуха перед телом может обеспечить снижение интенсивности ударной волны от тела. Это происходит из-за увеличения скорости звука и уменьшения числа Маха потока в тепловом следе. Положение области $Q 1$ относительно тела влияет на интенсивность ударных волн и их динамику. Одним из условий снижения уровня звукового удара является правильный выбор расстояния между областью нагрева $Q 1$ и телом [16]. Расчеты дальнего поля показали, что для снижения уровня звукового удара область нагрева потока должна располагаться на таких расстояниях от 

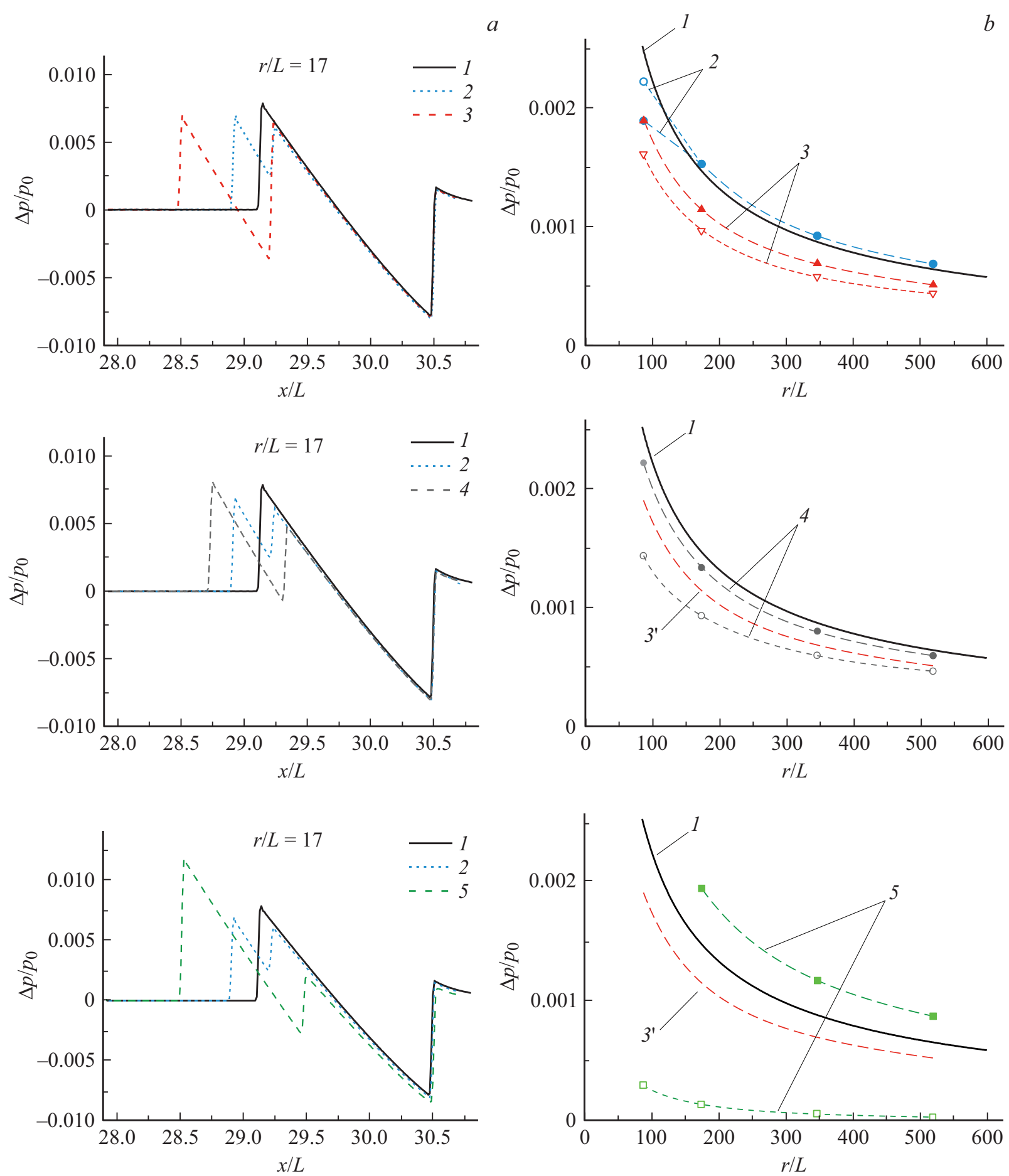

Рис. 5. Результаты расчетов для одной области нагрева потока $Q 1$. Профили избыточного давления в ближнем поле $(a)$ и интенсивность ударных волн в дальнем поле $(b): 1-$ тело в потоке без теплоподвода; $2-$ область нагрева с $r_{d} / L=0.05$, $x_{d} / L=0.0825$ и $T_{m} / T_{0}=1.93 ; 3$ - область нагрева с $r_{d} / L=0.05, x_{d} / L=0.5$ и $T_{m} / T_{0}=1.93 ; 3^{\prime}-$ интенсивность головной ударной волны от области нагрева с $r_{d} / L=0.05$ и $T_{m} / T_{0}=1.93$ (без тела); 4 - область нагрева $\mathrm{c} r_{d} / L=0.05, x_{d} / L=0.175$ и $T_{m} / T_{0}=2.41 ; 5$ - область нагрева с $r_{d} / L=0.1, x_{d} / L=0.175$ и $T_{m} / T_{0}=1.93$.

тела, при которых исключается слияние ударных волн от области нагрева и от тела в дальнем поле. Снижение уровня удара при раздельном распространении ударных волн показывают линии 3. В противном случае, слияние ударных волн в дальнем поле приведет к увеличению уровня звукового удара (линии 2). При увеличении температуры нагрева потока интенсивность ударной волны от области нагрева $Q 1$ растет (линии 4). Интенсивность ударной волны от тела, находящегося в тепловом следе, становится меньше интенсивности ударной волны от 
области нагрева. В этом случае звуковой удар будет определяться ударной волной от области нагрева потока, поэтому рост интенсивности ударной волны от области нагрева приведет к росту уровня звукового удара. Хотя вариант с более сильным нагревом потока и позволяет уменьшить уровень удара от тонкого тела примерно на 7\% (линии 4), это существенно хуже возможности уменьшения уровня удара на 20\%, которое было получено при меньшем нагреве в области $Q 1$ (линии 3 и $3^{\prime}$ ). К увеличению уровня звукового удара приводит и увеличение размера области нагрева (линии 5). Интенсивность ударной волны от области нагрева $Q 1$ становится больше интенсивности ударной волны от тела в холодном потоке.

\section{2. Разогрев набегающего потока с помощью нагретого диска перед телом}

Необходимо отметить, что рассматривалась и возможность разогрева набегающего потока с помощью разогрева диска, расположенного перед телом. Задача управления уровнем звукового удара с помощью диска, расположенного перед телом поперек набегающего потока, описана в работе [17]. На рис. 6 показана схема течения вблизи тела с диском. В области между телом 1 и диском 2 формируется возвратное дозвуковое течение (область аэродинамической тени). Границей области является звуковая линия 3. Ударная волна от тела возникает как висячий скачок 5 .

Выполнена серия расчетов, предполагающих разогрев потока с помощью нагретого диска. Параметры расчета: $L=40 \mathrm{~m}, p_{0}=5474.87 \mathrm{~Pa}, T_{0}=216.65 \mathrm{~K}$. Диаметр диска $0.04 L$. Диск расположен перед телом на расстоянии $0.046 L$ от носика тела. Для холодного диска задавалось условие отсутствия теплового потока через поверхность диска. Для нагретого диска температура поверхности диска задавалась равной 780 или $1560 \mathrm{~K}$. На рис. 7 показаны результаты расчетов при разогреве диска до температуры $1560 \mathrm{~K}$. Вычисленная температура дозвукового потока в области аэродинамической тени за холодным диском близка к температуре торможения набегающего потока $(390 \mathrm{~K})$ и составляла $T \sim 350-370 \mathrm{~K}$. Особенности возвратного течения за диском приводят к тому, что поток за нагретым диском разогревается

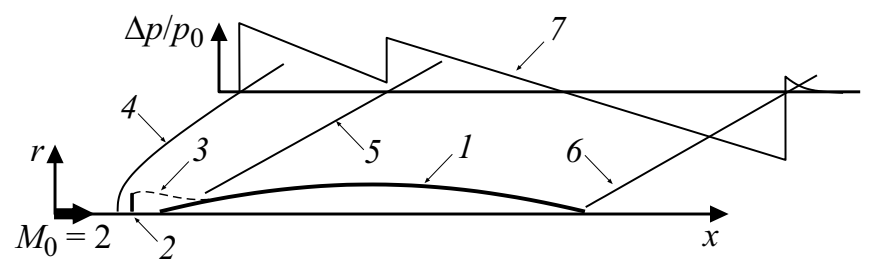

Рис. 6. Картина обтекания тела с диском: 1 - тонкое тело вращения, 2 - диск, 3 - звуковая линия, $4-$ ударная волна от диска, 5 - ударная волна от тела (висячий скачок), 6 - хвостовая ударная волна, 7 - профиль относительного избыточного давления.

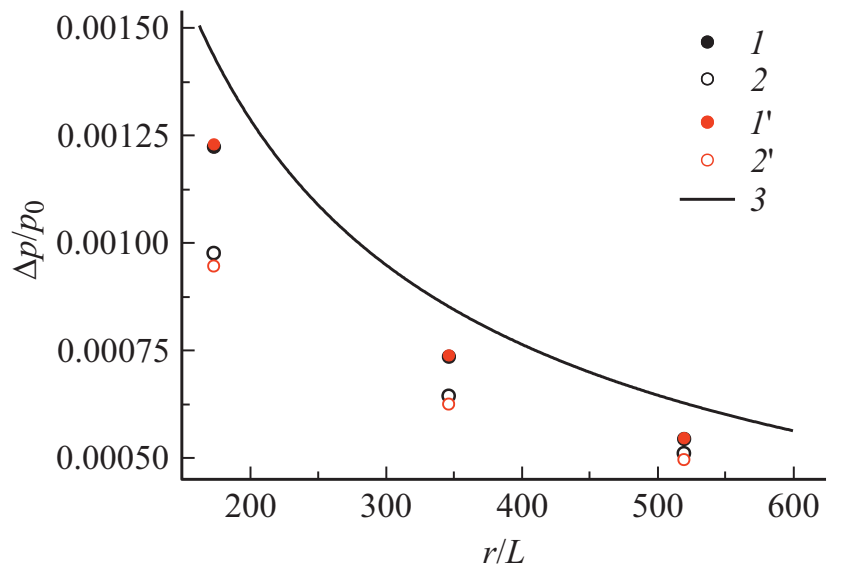

Рис. 7. Тело с нагретым диском $d / L=0.04 .1$ - относительная интенсивность ударной волны от холодного диска, 2 - относительная интенсивность висячего скачка от тела с холодным диском, $l^{\prime}$ - относительная интенсивность ударной волны от нагретого диска, $2^{\prime}$ - относительная интенсивность висячего скачка от тела с нагретым диском, 3 - относительная интенсивность головной ударной волны от тонкого тела без диска.

в среднем до температуры $T \sim 400-450 \mathrm{~K}$. Расчеты показали, что нагрев диска перед телом оказывает очень слабое влияние на картину течения в ближнем и дальнем поле. В рассмотренном случае объединение двух эффектов снижения уровня звукового удара за счет аэродинамической тени и увеличения скорости звука при разогреве потока не может быть реализовано при отсутствии проточности нагревательного элемента. Возможно, существует оптимальное соотношение между проточностью диска и температурой диска, которое обеспечит снижение уровня звукового удара.

\section{3. Пошаговый разогрев набегающего потока в двух областях перед телом}

Разогрев набегающего сверхзвукового потока в одной области, расположенной перед телом, может обеспечить уменьшение уровня звукового удара на 20\%. Более сильного эффекта можно достигнуть с помощью пошагового (последовательного) разогрева потока в нескольких областях перед телом.

На рис. 8 представлены результаты расчетов для двух областей нагрева $Q 1$ и $Q 2$, расположенных перед тонким телом. Размер областей нагрева $r_{d} / L=0.05$. Область нагрева $Q 1$ располагалась перед телом на расстоянии $x_{d} / L=0.5$, область нагрева $Q 2$ располагалась на расстоянии $x_{d} / L=0.9$. Поток нагревался в области $Q 2$ до наибольшей температуры $1.63 \cdot T_{0}$, в области $Q 1$ поток нагревался до температуры $2.8 \cdot T_{0}$ или до температуры $2.92 \cdot T_{0}$. Профили избыточного давления в ближнем поле показаны на рис. $8, a$ на расстоянии $r / L=17$ от траектории полета. На рис. $8, b$ показаны интенсивности ударных волн на больших расстояниях от траектории 

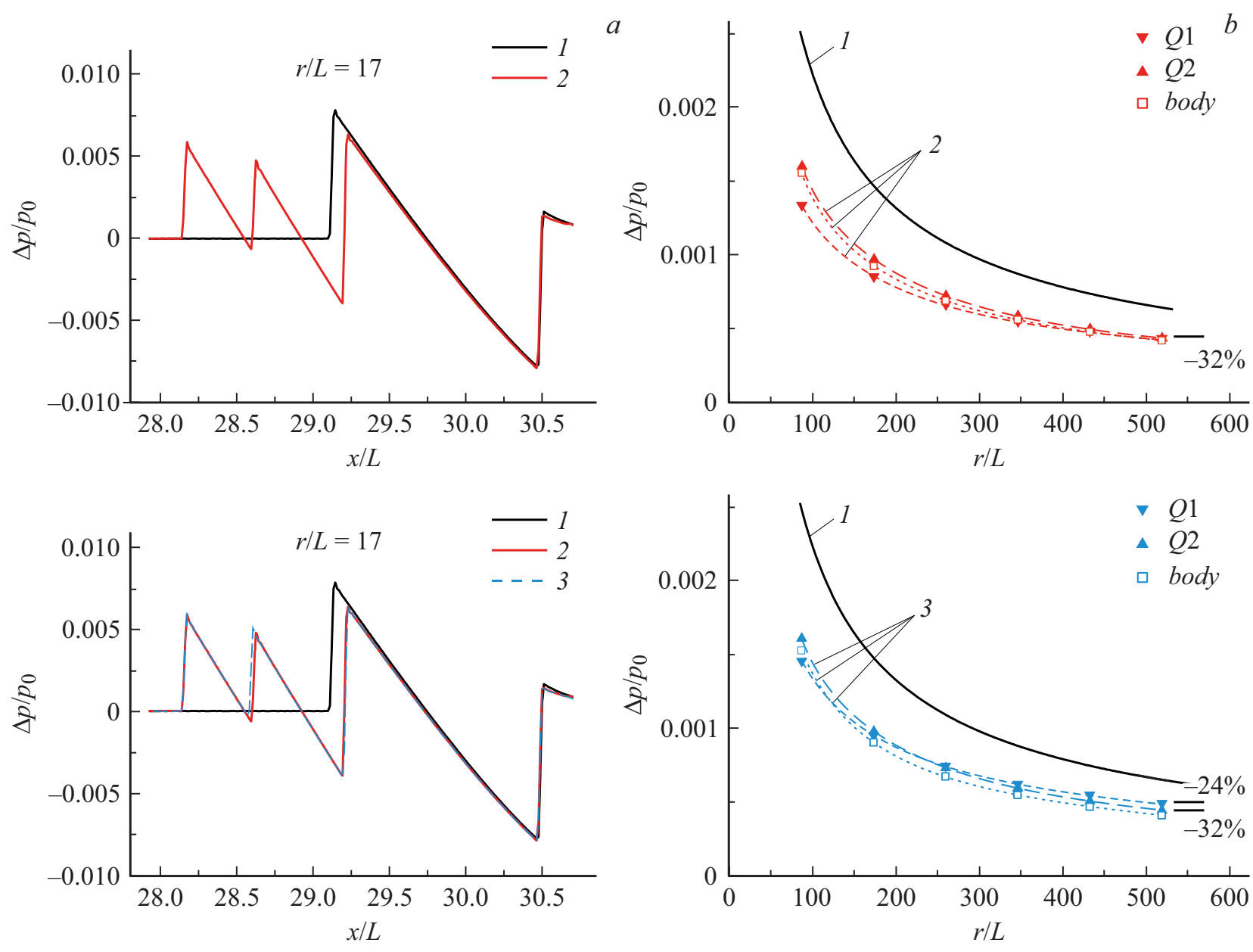

Рис. 8. Результаты расчетов для двух областей нагрева потока $Q 1$ и $Q 2$. Профили избыточного давления в ближнем поле $(a)$ и интенсивность ударных волн в дальнем поле $(b) .1$ - тело в потоке без теплоподвода, $2-$ область нагрева $Q 1$ с $T_{m} / T_{0}=2.8$ и область нагрева $Q 2$ с $T_{m} / T_{0}=1.63,3$ - область нагрева $Q 1$ с $T_{m} / T_{0}=2.92$ и область нагрева $Q 2$ с $T_{m} / T_{0}=1.63$, body интенсивности головной ударной волны от тела, находящегося в тепловом следе.

полета. Линия 1 показывает интенсивность головной ударной волны от тела в холодном потоке воздуха. Линии 2 и 3 показывают интенсивности ударных волн от тела и от областей нагрева потока. Сплошными точками показаны интенсивности ударных волн от областей нагрева $Q 1$ и $Q 2$, пустыми точками (body) интенсивности ударной волны от тела, находящегося в тепловом следе за областями нагрева. Горизонтальными линиями показаны уровни интенсивностей ударных волн от областей нагрева. Процентами показана разница между интенсивностями ударных волн и интенсивностью головной ударной волны от тела в холодном потоке на расстоянии $r / L \sim 520$. Правильный выбор расстояний между областями нагрева потока и телом обеспечил раздельное распространение ударных волн от тела и областей нагрева. При последовательном разогреве набегающего потока удалось уменьшить интенсивность ударных волн. Линиями 2 показаны результаты расчета, в котором удалось получить минимальные значения интенсивности ударных волн в дальнем поле. Интен- сивности всех ударных волн на расстоянии $r / L \sim 520$ примерно одинаковы. В этом случае разогрев потока в двух областях позволил снизить уровень звукового удара более чем на $30 \%$, по сравнению с ударом от тела в холодном потоке. Более сильный нагрев потока в области $Q 1$ приведет к росту интенсивности ударной волны, распространяющейся от этой области нагрева (линии 3). Интенсивность ударной волны от области $Q 2$ не меняется. Этот результат является менее предпочтительным, по сравнению с предыдущим. Незначительное увеличение температуры в области $Q 1$ (менее 5\%) приводит к тому, что на расстояниях $r / L>260$ интенсивность ударной волны от области нагрева $Q 1$ становится больше интенсивности ударных волн от области $Q 2$ и тела, находящегося в тепловом следе. На расстояниях $r / L>520$ ударные волны от $Q 1$ и $Q 2$ сольются, что приведет к увеличению уровня звукового удара.

Способ уменьшения уровня звукового удара при последовательном локальном разогреве набегающего потока в нескольких областях представляется перспектив- 
ным. В то же время при увеличении количества областей нагрева сложная картина распространения большого числа слабых ударных волн и необходимость явного их выделения в расчетах создает огромные трудности в задаче прогноза звукового удара. В настоящее время представляется затруднительным проведение расчета дальнего поля течения с большим количеством областей нагрева.

\section{Заключение}

Представлены результаты численного решения задачи о звуковом ударе от тонкого тела в сверхзвуковом набегающем потоке воздуха при различных способах локального нагрева набегающего потока. Расчеты выполнены с помощью комбинированного численно-аналитического метода „тел-фантомов“ при числе Маха набегающего потока воздуха равного 2.

Показано, что разогрев набегающего сверхзвукового потока в одной области, расположенной перед телом, уменьшает уровень удара примерно на 20\% по сравнению с ударом от тела в холодном потоке. Попытка улучшения результата при более сильном разогреве набегающего потока только в одной области теплоподвода приведет к усилению звукового удара, поскольку ударная волна от области нагрева при высоких температурах будет усиливаться и может иметь интенсивность, превышающую интенсивность ударной волны от исходного тела в холодном набегающем потоке. Разогрев набегающего на тонкое тело потока с помощью нагрева диска, расположенного перед телом поперек потока, оказывает очень слабое влияние на картину течения в дальнем поле.

Разогрев потока в двух областях позволяет снизить уровень звукового удара более чем на $30 \%$ по сравнению с ударом от тела в холодном потоке.

Результаты работы позволяют утверждать, что пошаговый разогрев набегающего потока в нескольких последовательно расположенных областях обеспечит существенное снижение уровня звукового удара, по сравнению с ударом от тела в холодном потоке.

\section{Финансирование работы}

Работа выполнена в рамках Программы фундаментальных научных исследований государственных академий наук на 2013-2020 годы (проект АААА-А17117030610126-4).

\section{Конфликт интересов}

Авторы заявляют, что у них нет конфликта интересов.

\section{Список литературы}

[1] D.S. Miller, H.W. Carlson. A study of the application of heat or force fields to the sonic-boom-minimization problem. TN D-5582 (NASA, Washington, 1969)

[2] Н.А. Герасимов, В.С. Сухомлинов. ЖТФ, 80 (1), 34 (2010). [N.A. Gerasimov, V.S. Sukhomlinov. Tech. Phys., 55 (1), 33 (2010). DOI: 10.1134/S1063784210010068]

[3] Н.А. Герасимов, В.С. Сухомлинов. ЖТФ, 80 (11), 6 (2010). [N.A. Gerasimov, V.S. Sukhomlinov. Tech. Phys., 55 (11), 1553 (2010). DOI: 10.1134/S1063784210110022]

[4] J.K. Riley. Sonic boom attenuator (US Patent 5,263,661. 1993)

[5] S.H. Zaidi, M.N. Shneider, R.B. Miles. AIAA J., 42 (2), 326 (2004). DOI: $10.2514 / 1.9097$

[6] Y. Sun, H. Smith. Prog. Aerosp. Sci., 90, 12 (2017). DOI: $10.1016 /$ j.paerosci.2016.12.003

[7] А.В. Потапкин, Ю.Н. Юдинцев. Уч. записки ЦАГИ, 14 (4), 26 (1983).

[8] A.V. Potapkin, T.A. Korotaeva, D.Yu. Moskvichev, A.P. Shashkin, A.A. Maslov, J.S. Silkey, F.W. Roos. An advanced approach for the far-field sonic boom prediction (AIAA Paper, 2009-1056, 2009). DOI: 10.2514/6.2009-1056

[9] А.В. Потапкин, Д.Ю. Москвичев. ПМТФ, 52 (2), 15 (2011). [A.V. Potapkin, D.Yu. Moskvichev. J. Appl. Mech. Tech. Phys., 52 (2), 169 (2011). DOI: 10.1134/S0021894411020027]

[10] R. Yamashita, K. Suzuki. AIAA J., 54 (10), 3223 (2016). DOI: $10.2514 / 1 . J 054581$

[11] Л.Д. Ландау. ПММ, 9 (4), 286 (1945).

[12] G.B. Whitham. Commun. Pure Appl. Math., 5 (3), 301 (1952). DOI: $10.1002 /$ cpa.3160050305

[13] G.B. Whitham. J. Fluid Mech., 1 (3), 290 (1956). DOI: $10.1017 / \mathrm{S} 0022112056000172$

[14] P.S. Rao. Aeronaut. Q., 7 (1), 21 (1956). DOI: $10.1017 / \mathrm{S} 0001925900010118$

[15] W.D. Hayes, R.C. Haefeli, H.E. Kulsrud. Sonic boom propagation in a stratified atmosphere, with computer program. CR - 1299 (NASA. Washington, 1969)

[16] A.V. Potapkin, D.Yu. Moskvichev. Shock Waves, 24 (4), 429 (2014). DOI: 10.1007/s00193-014-0503-x

[17] A.V. Potapkin, D.Yu. Moskvichev. Shock Waves, 28 (6), 1239 (2018). DOI: 10.1007/s00193-018-0817-1

[18] С.К. Годунов. Численное решение многомерных задач газовой динамики (Наука, М., 1976)

[19] А.П. Красильщиков, Л.П. Гурьяшкин. Экспериментальные исследования тел вращения в гиперзвуковых потоках (Физматлит, М., 2007) 